\title{
Autonomía y evaluación para la transformación de las prácticas educativas
}

\author{
Autonomy and Evaluation for the Transformation of Educational Practices
}

\author{
Adriana Estrada-Girón ${ }^{a}$, Amelia Molina-García ${ }^{b}$
}

\begin{abstract}
:
This essay analyzes some challenges of the assessment process by competences, its relationship with the concept of educational quality and the possibility of building autonomy in students, particularly in the field of higher education. It is assumed that evaluation in the classroom is a key element to improve the quality of education, not in a linear sense, but as the basis for the construction of a reflective process through which autonomy is built and democratic participation helps school processes, because this type of participation constitutes a reference that regulates behaviors, thinking and acting of the different educational actors.
\end{abstract}

\section{Keywords:}

Evaluation, evaluation by competences, educational quality, autonomy

\section{Resumen:}

Este ensayo analiza algunos retos del proceso de evaluación por competencias, su relación con el concepto de calidad educativa y la posibilidad de construcción de la autonomía en estudiantes, de manera particular en el ámbito de la educación superior. Se asume que la evaluación en el aula es un elemento clave para mejorar la calidad educativa, no en un sentido lineal, sino como base para la construcción de un proceso reflexivo a través del cual se forma la autonomía y se favorece la participación democrática en los procesos escolares, pues este tipo de participación constituye un referente que regula modos de ser, pensar y de actuar en los distintos actores educativos.

\section{Palabras Clave:}

Evaluación, evaluación por competencias, calidad educativa, autonomía

Recibido: 04 de junio de 2018

Dictaminado: 16 de agosto de 2018

Segunda versión: 4 de septiembre de 2018

Aceptado: 27 de septiembre de 2018

\footnotetext{
a Estudiante del Programa de Doctorado en Ciencias de la Educación. Universidad Autónoma del Estado de Hidalgo. Pachuca de Soto, Hidalgo: México, ORCID: https://oricid.org/0000-0002-1071-8142 Email: adriana.met.uaeh@gmail.com

b Profesora Investigadora del Área Académica de Ciencias de la Educación de la Universidad Autónoma del Estado de Hidalgo. Pachuca de Soto, Hidalgo: México. ORCID: https://oricid.org/0000-0002-8268-8421 Email:: molinag@uaeh.edu.mx
} 


\section{Introducción}

El presente ensayo tiene como propósito analizar algunos aspectos clave en torno a la evaluación por competencias, partiendo del supuesto de que este tipo de evaluación posee una gran relevancia dentro del proceso de formación integral de los estudiantes y la conformación de la autonomía. Por otro lado, también se pretende examinar algunos de los retos que deben enfrentar las instituciones escolares para llevar a cabo una tarea tan compleja como ésta, todo ello vinculado al concepto de calidad educativa. Con base en ello, se reflexionará sobre la importancia de la evaluación en estudiantes de nivel superior para el desarrollo de conductas autonómicas, asumiendo que la evaluación es fundamental para atender a la necesidad de mejorar no sólo la calidad de la educación que se imparte en las aulas, sino la de formar ciudadanos participativos y autónomos, considerando que: "La autonomía se va constituyendo en la experiencia de varias e innumerables decisiones que van siendo tomadas..." (Molina, 2016:139), entre estas experiencias se encuentran como preeminentes las que se viven en la escuela, construidas a través de los procesos que se dan al interior, entre los que se encuentra el de la evaluación. Considerando que el "[...] desarrollo de la autonomía significa llegar a ser capaz de pensar por sí mismo con sentido crítico, teniendo en cuenta puntos de vista diferentes al personal, tanto en el ámbito moral como en el intelectual" (Molina, 2017:9).

Para alcanzar el objetivo planteado, se retomarán algunos conceptos clave, tales como: evaluación, modelo basado en competencias, evaluación por competencias y calidad, esto con el objetivo de centrar la discusión sobre los retos de la evaluación por competencias con base en referentes conceptuales vigentes en la temática. Posteriormente, se abordan los retos de la evaluación por competencias y su relación con la participación democrática y el desarrollo de la autonomía.

\section{Evaluación y calidad en la educación}

De acuerdo con Moreno (2012), la evaluación es un elemento central en el proceso formativo, con implicaciones en todos los agentes educativos y en el Sistema Educativo Nacional (SEN). Por otra parte, constituye un elemento esencial, a partir del cual es posible obtener información sobre cómo se va desarrollando cualquier proceso formativo y sobre la calidad de los aprendizajes efectivos de los estudiantes. Desde esta lógica cabría entonces preguntarse ¿qué aprendizajes habría que evaluar?, esta pregunta, nos remite necesariamente al modelo bajo el cual deseamos llevar a cabo la evaluación. Para Moreno (2009:565) la evaluación del aprendizaje se ha ido transformando, desde concepciones tradicionales vinculadas "con el concepto de medida del rendimiento académico de los contenidos disciplinares (valoración de los resultados), manteniendo una visión examinadora y de control, y restringida al aprendizaje de los alumnos" hacia una mirada más amplia, desde la que se considera la existencia de factores que no necesariamente son cuantificables. Desde el enfoque basado en competencias, los resultados de aprendizaje se refieren al desempeño observable de los estudiantes en distintos niveles: conceptual, procedural y actitudinal, mismos que deben reflejar lo que se espera que el estudiante conozca, aprehenda, analice y exprese, durante y especialmente al término de un proceso de enseñanza (Villarroel y Bruna, 2014). Desde esta perspectiva, el enfoque por competencias "amplía el sentido de escolaridad y lo vincula a las prácticas sociales y a la vida" (Moreno, 2012:2), las metodologías cambian, entonces, desde una visión basada en la enseñanza a una centrada en el aprendizaje.

En contraste con lo anterior, es importante tener en cuenta, como menciona Freire (1996:42), que "no puede existir una práctica educativa neutra, no comprometida, apolítica. La directividad de la práctica educativa que la hace trascenderse siempre a sí misma y que la hace perseguir un determinado fin, un sueño, una utopía, no permite su neutralidad".

Siguiendo a Freire, la práctica educativa es politizada, por consiguiente sustentada en intereses e ideas de un momento y contexto histórico-social determinado, lo mismo ha sucedido con el concepto de calidad en educación, el cual se ha construido y reconstruido a partir de múltiples dimensiones, entre ellas: la ideológica, la social, la política y la histórica. Siendo así, calidad entonces se convierte en un concepto transmutable, dependiente de las ideas acerca de lo que debería de ser la mejor educación, cómo deberá proporcionarse y a quiénes, esto para llegar al ideal de ciudadano y sociedad que se desean alcanzar, debido a ello resulta ser un concepto no estático y relativo (Escudero, 2003; Miranda y Miranda, 2012; Santos, 1999).

En complemento con lo anterior, debemos reconocer la índole política de la educación, su falta de neutralidad, con ello podremos hablar de ética y de un ejercicio democrático, en virtud de que la práctica educativa y la formación humana, al no ser neutras implican: "opciones, rupturas, decisiones, estar y ponerse en contra, en favor de un sueño y contra otro, en favor de alguien y contra alguien" (Freire, 1996:43). 
Sin negar la falta de neutralidad de la educación y por consiguiente del concepto de calidad ligado a ella, en lo que respecta a este último, se han presentado diversos momentos donde se ha puesto énfasis en aspectos diferentes $y$, tal vez, hasta contradictorios. Sin embargo, lo que no debe perderse de vista es que la calidad de la educación deberá ser vista como una exigencia de justicia y equidad social, por lo que habrá de articularse el Sistema Educativo como un todo -con conocimiento, formación, recursos materiales, aspectos morales, entre otros relacionados con los procesos de formación y de gestión de la educación- lo que deberá apuntar a una mejora de los procesos formativos, entre ellos la evaluación y por consiguiente, la formación de ciudadanos autónomos y participativos.

Por otra parte, para poder hacer referencia a los desafíos de la evaluación por competencias, será necesario reflexionar acerca de las reformas educativas vigentes en nuestro país, que si bien, se han llevado a cabo en los niveles básicos, también tienen un impacto sobre la educación superior. Al respecto, Flores y García (2014) afirman que las instituciones de educación superior (IES) deben prestar atención a lo que ocurre en niveles educativos precedentes, por tres razones:

Primero, porque la reforma toca la manera de operar de los bachilleratos públicos; segundo, porque en el nivel universitario hace falta una noción de calidad mejor construida; tercero, porque las IES pueden ampliar sus capacidades de vigilancia crítica sobre las acciones que propone el gobierno en materia educativa. Las universidades públicas autónomas poseen la capacidad para mostrar independencia y libertad de crítica (Flores y García, 2014: 9).

Las reformas educativas en nuestro país, han sido producto de políticas y acuerdos internacionales, pero también, como lo afirman Villarroel y Bruna (2014), han sido derivadas de la presión desde distintos ámbitos de la sociedad, en los que se generó discusión respecto a la calidad de la educación que se estaba ofreciendo en las aulas. No obstante, la realidad ha mostrado que las reformas han sido insuficientes para mejorar la calidad de la educación y, en buena medida, se debe a factores como la falta de un marco teórico sólido que dé sustento y clarifique el enfoque basado en competencias; a la vaguedad y carencia de referentes conceptuales, lo que dificulta la operación y da lugar a vacíos metodológicos que obstaculizan no sólo la construcción de aprendizajes sino también la evaluación; a la insuficiencia de materiales curriculares para apoyar a los docentes y su escasa difusión, finalmente a propuestas de formación docente poco sólidas y superficiales que no favorecen en los docentes el desarrollo competencias profesionales, necesarias para atender eficazmente lo que postulan las reformas actuales y venideras (Moreno, 2012; Moreno, 2011).

Una reforma, como lo mencionan Flores y García (2014), implica el establecimiento de "nuevas reglas", dentro de ellas no debemos perder de vista que todos los niveles educativos deberán articularse, las IES no están al margen de estos cambios en diversos aspectos, entre ellos la construcción de la noción de calidad. Sin embargo, calidad entonces, es un constructo que cuando intenta traducirse en reformas y propuestas curriculares concretas y pertinentes resulta "volátil", es decir, cuando se intenta llevar a la práctica puede "evaporarse" pero... ¿qué se pretende decir con esto?

Casanova (2012:15) menciona que las reformas en nuestro país, "no se llevan a cabo con la profundidad que exige el momento histórico actual ni la sociedad que nos rodea". Esto es, de acuerdo con la autora, seguimos manteniendo modelos tradicionales en las estructuras curriculares con formas de evaluación poco pertinentes para las necesidades que nos plantea la realidad tan compleja en la que se da el hecho educativo, lo que se traduce, a su vez, en los pobres resultados que presenta nuestro sistema educativo cuando lo contrastamos con referentes internacionales. Suponiendo que cumplir con indicadores, para los cuales se utilizan los resultados de las evaluaciones, es un referente de calidad, cuando en lo particular se le está mirando más como un requisito de cumplimiento de aspectos administrativos y de índole diferente a aquello que implica centrarse en los procesos de apropiación y desempeño de los estudiantes en formación y, como parte de su ejercicio profesional. Es decir, que tengan la competencia para dar respuesta a lo que se requiere del ejercicio de su profesión, llevando este proceso reflexivo.

Hasta aquí, tenemos entonces dos posturas, aparentemente contradictorias, de las cuales es posible hacer un análisis más profundo, sin embargo, al no ser el objetivo de este trabajo, solo se dejarán expuestas. Por un lado, tenemos claro que los cambios en los modelos curriculares derivados de las reformas y todo lo que implican en los distintos procesos que se dan en el hecho educativo, entre ellos la evaluación, no son neutros, pues responden a las necesidades planteadas por el contexto histórico, así como a intereses políticos y sociales. Pero, por otra parte, está el hecho de la resistencia al cambio, esa resistencia que se mantiene en formas tradicionales de llevar a cabo la práctica educativa y, como parte de esta práctica, la evaluación. 


\section{Retos de la evaluación por competencias}

Con base en lo anterior, podemos decir, que la no neutralidad de la educación, nos ha llevado a transitar a modelos como el de competencias. No obstante, las reformas $y$, en el nivel superior, las propuestas curriculares que pretenden dar respuesta a las actuales demandas, tenemos aún muchos retos por afrontar, el proceso de evaluación con todo lo que involucra es uno de ellos.

\section{Primer reto: vencer las resistencias}

Un primer reto importante para la evaluación, implica vencer las resistencias, ya que "si [hay que considerar algo] dentro de la cultura de la escuela [es que] existe una dimensión que revela mejor la existencia de creencias, mitos, ritos, etc., que se resisten al cambio, esa dimensión es la evaluación" (Moreno, 2011:120). Por lo que es necesario fomentar cambios en la concepción tradicional de evaluación, que aún está presente en algunas de las aulas, y que se encuentra al margen de los planteamientos del aprendizaje basado en competencias. Esto requiere hacer a un lado hábitos arcaicos, métodos y procedimientos de evaluación que han sido rebasados por las propuestas pedagógicas actuales.

\section{Segundo reto: vinculación con el proceso de enseñanza-aprendizaje}

Al centrarnos en la evaluación por competencias, debemos tener en cuenta que los resultados del Sistema Educativo Nacional, muestran que escuela, lejos de acortar la brecha entre los distintos grupos sociales ha tendido a profundizarla (Moreno, 2011). Si vemos a la evaluación como una herramienta para mejorar la calidad educativa, una parte importante de esta complicada tarea está justamente en la evaluación de las competencias en el aula. Entonces, otro de los retos de esta evaluación es que deberá presentar una fuerte vinculación con el proceso de enseñanza y aprendizaje, atendiendo no sólo al resultado final derivado de éste, sino a todo el desarrollo. Para ello se deben considerar los diversos tipos de evaluación: diagnóstica, formativa, sumativa, coevaluación y autoevaluación.

Atendiendo a lo anterior, el docente deberá plantearse las cuestiones fundamentales de todo proceso de evaluación: ¿Para qué y cómo evaluar? ¿Qué funciones cumple la evaluación que realizan? (Moreno, 2009; Casanova 2011). Considerando, al mismo tiempo, que la evaluación por competencias tiene que ser, un juicio integral, resultado de las diversas evaluaciones formativas cualitativas, lo cual no está exento de dificultad, pues, la evaluación debe llevar a una reflexión de docentes y estudiantes, ya que mediante esta actividad se integra la información de todo el proceso de aprendizaje y se activan cuestiones que les permitirán llegar a una transformación que amplíe en ambos la visión del mundo, con una mirada más analítica, que fomente la autocrítica, la participación democrática y les permita trasladar lo aprendido a diversos contextos, con todo ello estaremos favoreciendo la formación de ciudadanos autónomos.

\section{Tercer reto: el uso de los resultados de evaluación}

La evaluación de los aprendizajes tendrá que servir para algo más que acreditar una asignatura. Deberá, como se mencionó, propiciar la autocrítica a partir de la reflexión, pero además, proveer elementos que lleven al estudiante a transitar hacia la autorregulación de su propio proceso de aprendizaje; aspectos clave de un modelo que, como el de competencias, está centrado en el aprendizaje, donde es el estudiante y no el docente el protagonista de este proceso. Los resultados de los procesos de evaluación deberán permitir al estudiante tener una base de información amplia para tomar decisiones en los ámbitos profesional y personal con sentido crítico y responsabilidad, aspectos clave para el desarrollo y conformación de la autonomía.

\section{Cuarto reto: concientización sobre la importancia de la autoevaluación y la co- evaluación}

La autoevaluación y la co-evaluación son herramientas fundamentales para fomentar la autocrítica y una actitud reflexiva permanente en los estudiantes, considerando que en ambas se pone en juego no sólo competencias disciplinares o conocimientos, sino que van más allá, pues reflejan actitudes y valores. Por ello, una gran reto al emplearlas es el de concientizar a quienes las realizarán sobre la importancia de valorar a sus pares y a sí mismos de manera justa, honesta y objetiva, teniendo en cuenta que estas evaluaciones les permiten una participación democrática en su proceso formativo, lo que implica un ejercicio responsable y desde el respeto activo para con el otro (Cortina, 2001).

De acuerdo con Padilla y Gil (2008) este tipo de evaluación deberá aportar criterios y estándares claros que permitan comprender al estudiante lo que se requiere en una actividad, y con ello, podrán alcanzar mejores resultados, no sólo en términos de evaluación sino de aprendizajes. Además de esto, desde lo 
planteado por los autores, es necesario implicar a los estudiantes en la formulación de estos criterios, pues esto mejorará la comprensión y favorece el proceso de aprendizaje y, desde la perspectiva manejada en este trabajo, favorece la autonomía.

Con base en lo expuesto en el párrafo anterior, cabría preguntarse ¿de qué forma la autoevaluación y la coevaluación pueden favorecer el desarrollo de la autonomía en los estudiantes? Para responder a este planteamiento, es importante considerar que una de las tareas fundamentales de la escuela es la de promover la autonomía, en el ámbito socio-moral, esto involucra el reconocimiento de que tal pretensión no se logra de manera natural, ni es consecuencia lógica del desarrollo, se trata de un proceso que lleva a los individuos a trasladarse de forma gradual de un plano heterónomo, donde los criterios para valorar lo justo se encuentran depositados en el exterior, en los otros, hacia uno autónomo, en el que los comportamientos justos se presentan por convicción, con independencia de los otros, pero sin dejar de tenerlos en cuenta (Landeros y Chávez, 2015).

La evaluación genera tensiones y choques de intereses y valores. En este sentido, la participación dentro del proceso de evaluación propio y de los pares es una oportunidad para permitir a los estudiantes expresar una multiplicidad de concepciones con respecto al proceso y con relación a lo que realmente consideran que aprendieron, a su visión sobre lo que es justo que sus pares y ellos obtengan como resultado de su trabajo. Todo ello, mediante un proceso reflexivo, esto es, la evaluación resulta valiosa para construir autonomía, refiriéndonos a ella a partir de la teoría del desarrollo moral de Kohlberg (1992), donde la autonomía moral es la capacidad de elegir cómo conducirse ante ciertas situaciones a partir de una elección propia dimensionada con referentes construidos en relación con "los otros". Es decir, no se deja de tomar en cuenta lo que socialmente es "aceptable", sin embargo, las elecciones se hacen por convicción y no por obtener aprobación (Kohlberg, Power \& Higgins, 2008).

\section{Quinto reto: el docente como agente reflexivo en el proceso de evaluación}

La responsabilidad del docente, dentro del proceso de formación de los estudiantes, involucra más de una tarea, entre ellas, se encuentra la de comprobar y valorar el grado de aprendizaje de cada uno de los estudiantes, en el nivel superior, lo que debe orientarse al aseguramiento del éxito académico y posteriormente profesional de ellos (De la Mano y Moro, 2009). Por lo que formar o desarrollar competencias requiere de capacitación, pero esto no es suficiente, debe involucrar "un continuo aprender haciendo reflexivo", donde el docente deberá tomar consciencia de los logros y errores propios para implementar acciones correctivas en la práctica docente (Tobón, 2006). Siguiendo esta idea, el profesor también deberá tener en cuenta que cuando enseña y evalúa promueve valores, actitudes, modos de actuar, induce incluso, formas de pensar, por lo que se vuelve un modelo para sus estudiantes, bajo esta lógica, deberá llevar a cabo una evaluación reflexiva, que genere las condiciones para que a partir de ello los estudiantes puedan explotar su potencial, ser participantes activos, reflexivos, críticos con su proceso y, derivado de ello, desarrollar autonomía.

\section{Sexto reto: la inclusión}

Retomando el concepto de participación democrática, se analizará un último reto, el de la inclusión. Molina (2015) reporta que en los últimos años han surgido, en diversos países, movimientos estudiantiles que confirman su disposición hacia la participación y el interés por los asuntos que les afectan, entre ellos: asuntos públicos, la justicia social y la inclusión de los grupos minoritarios. En tal sentido, en una sociedad democrática la educación debe ser necesariamente inclusiva, de otra forma, no cumpliría con la función de formar ciudadanos "que deberán participar activa, creativa y críticamente y en la creación del futuro de su nación o grupo de pertenencia" (Casanova, 2011:79). Atender el aspecto de la inclusión implica, respetar los estilos y ritmos de aprendizaje, las diferencias en las capacidades cognitivas de los estudiantes y por supuesto, tener en cuenta el contexto en el cual los estudiantes se desenvuelven. Parece que esto empieza a ser más claro en lo concerniente a la construcción de los aprendizajes, no así, en lo que respecta a la forma de evaluarlos, pues habría que diversificar también las estrategias para atender a las particularidades y de esta forma poder valorar con objetividad lo que el estudiante ha conseguido.

\section{Conclusiones}

Es importante señalar que los retos abordados, hasta el momento en este ensayo, son solo algunos de los muchos que existentes en lo que respecta a la evaluación por competencias. Es esencial tener en cuenta que la evaluación es una poderosa herramienta para lograr cambios favorables, pero para ello será necesario que dentro del espacio escolar se atienda a sus funciones pedagógicas y de formación, antes que a cuestiones de tipo administrativo y de control. La evaluación debe asegurar que los docentes y estudiantes obtengan información confiable y pertinente acerca del 
proceso de enseñanza y aprendizaje, además de utilizarla para conseguir mejorar, si se desea emplear la evaluación para lograr educación de calidad. Debemos ocuparnos entonces de que la evaluación, con todo lo que implica, planeación, objetivos, técnicas, uso de resultados, etc., ofrezca y presente también calidad.

Aunado a lo anterior, no debemos perder de vista que en la evaluación por competencias, tenemos una poderosa herramienta para promover la participación democrática y el desarrollo de la autonomía. La evaluación deberá ser planteada de tal forma que, además de brindar información acerca de lo que el estudiante ha aprendido, les permita reflexionar de una manera crítica integrando diferentes perspectivas: la suya, la de sus pares, la de sus docentes y, de esta forma, transitar de un plano heterónomo a uno autónomo.

La autonomía guarda relación con una serie de valores, donde tal relación es bidireccional. Desde los valores se construye autonomía, pero al mismo tiempo, permite ponerlos en práctica y cuestionar su ejercicio. La evaluación es una oportunidad para construir esta compleja capacidad, donde cada estudiante involucra reflexión, evalúa sus opciones, las jerarquiza y, al mismo tiempo, pone en juego valores desde los cuales va a interactuar no sólo en el espacio escolar, sino también en otros contextos.

\section{References}

Casanova, María Antonia (2011). "Evaluación para la Inclusión Educativa". Revista Iberoamericana de Evaluación Educativa, 4(1), 79-89.

Casanova, María Antonia (2012). "El diseño curricular como factor de calidad educativa". Revista Iberoamericana sobre Calidad, Eficacia y Cambio en Educación, 10(4), 6-20.

Cortina, Adela (2001). Ciudadanos del mundo. Hacia una teoría de la ciudadanía. Madrid, España: Alianza editorial.

De la Mano, Marta y Moro, Manuela (2009). La evaluación por competencias: propuesta de un sistema de medida para el grado en Información y Documentación [en línea]. bid textos universitaris de biblioteconomia $i$ documentació, No. 23. Barcelona: Facultat de Biblioteconomia i Documentació Universitat de Barcelona. Disponible en: http://bid.ub.edu/23/delamano2.htm [30 de septiembre de 2018]

Escudero, Juan Manuel (2003). "La calidad de la educación: controversias y retos para la educación pública”. Education (20-21), 21-38.

Flores, Pedro y García, César (2014). "La Reforma Educativa en México. ¿Nuevas reglas para las IES? Revista de la Educación Superior, ANUIES, XLIII-4(172), 9-31.

Freire, Paulo (1996). "Educación y calidad". En P. Freire, Política y educación (págs. 41-49). México: Siglo XXI.
Kohlberg, Lawrence (1992). Psicología del desarrollo moral. España; Desclée de Brouwer.

Kohlberg, Lawrence, Power, F. Clark \& Higgins, Ann (2008). La Educación Moral según Lawrence Kolhberg. Barcelona: Gedisa.

Landeros, Leticia y Chávez, Concepción (2015). "Convivencia y disciplina en la escuela. Análisis de reglamentos escolares de México" [En línea] Ciudad de México: Instituto Nacional para la Evaluación de la Educación. Disponible en: http://publicaciones.inee.edu.mx/buscadorPub/P1/C/232/P1C232.pdf [30 de enero de 2018]

Miranda, José Francisco y Miranda, Jesús Bernardo (2012). "Reflexiones sobre la calidad de la educación y sus referentes: el caso de México". Educere, 16(53), 43-52.

Molina, Amelia (2015). "Espacios alternativos de formación en la construcción de culturas políticas: una propuesta para su análisis". Revista de Prácticas y Discursos. Cuadernos de Ciencias Sociales, $4(4), 1-25$.

Molina, Amelia (2016). "El papel de la ética en las ciudadanías emergentes: autonomía y derechos humanos como referentes de reflexión para un proceso de investigación". En T. Yurén, L. M. Ibarra, \& A. E. Escalante, Investigación en Educación y Valores: Ética, ciudadanía y Derechos Humanos. Morelos, México: Universidad Autónoma del Estado de Morelos.

Molina, Amelia, Ramos, Gerardo y Sáenz, J. Antonio (2017). "La autonomía en la práctica de los docentes de nivel secundaria: un estudio exploratorio sobre sus percepciones y autopercepciones" [En línea]. México: Congreso Mexicano de Investigación Educativa. Disponible http://www.comie.org.mx/congreso/memoriaelectronica/v14/doc/2719. pdf [30 de junio de 2018].

Moreno, Tiburcio (2009). "La evaluación del aprendizaje en la universidad". RMIE, 14(41), 563-591.

Moreno, Tiburcio (2011). "La cultura de la evaluación y la mejora de la escuela". Perfiles Educativos, 33(131), 116-130.

Moreno, Tiburcio (2012). "La evaluación de competencias en educación”. Sinéctica (39)1-20.

Padilla, M. Teresa y Gil, Javier (2008). "La evaluación orientada al aprendizaje en la Educación Superior: condiciones y estrategias para su aplicación en la docencia universitaria". Revista española de Pedagogía. Año LXVI, no. 241, Disponible en: https://dialnet.unirioja.es/servlet/articulo?codigo=2709011 $\quad\left[\begin{array}{ll}15 & \text { de }\end{array}\right.$ agosto de 2018].

Santos, Miguel Ángel (1999). "Las Trampas de la Calidad". Acción pedagógica, 8(2), 78-81.

Tobón, Sergio (2006). "Formación Basada en Competencias" [en Línea]. Madrid, España: Universidad Complutense de Madrid. Disponible en: https://www.uv.mx/psicologia/files/2015/07/Tobon-S.-Formacionbasada-en-competencias.pdf [30 de septiembre de 2018].

Villarroel, Verónica., \& Bruna, Daniela (2014). "Reflexiones en torno a las competencias genéricas en educación superior: Un desafío pendiente". Psicoperspectivas, 13(1), 23-34. 